\title{
PMNs facilitate translocation of platelets across human and mouse epithelium and together alter fluid homeostasis via epithelial cell-expressed ecto-NTPDases
}

Thomas Weissmüller, ${ }^{1,2}$ Eric L. Campbell,1 Peter Rosenberger, ${ }^{2}$ Melanie Scully, ${ }^{1}$ Paul L. Beck, ${ }^{3}$ Glenn T. Furuta, ${ }^{1,4}$ and Sean P. Colgan'1

\begin{abstract}
${ }^{1}$ Mucosal Inflammation Program, Division of Gastroenterology, University of Colorado Denver School of Medicine, Aurora, Colorado, USA. 2Department of Anesthesiology and Intensive Care Medicine, Tübingen University Hospital, Tübingen, Germany. ${ }^{3}$ Division of Gastroenterology Gastrointestinal Research Group, University of Calgary, Calgary, Alberta, Canada. ${ }^{4}$ Section of Gastroenterology, Hepatology and Nutrition, The Children's Hospital, Aurora, Colorado, USA.
\end{abstract}

\begin{abstract}
Mucosal diseases are often characterized by an inflammatory infiltrate that includes polymorphonuclear leukocytes (PMNs), monocytes, lymphocytes, and platelets. A number of studies have suggested that the interaction of platelets with leukocytes has an essential proinflammatory role. Here, we examined whether platelets migrate across mucosal epithelium, as PMNs are known to do, and whether platelets influence epithelial cell function. Initial studies revealed that human platelets did not efficiently transmigrate across human epithelial cell monolayers. However, in the presence of human PMNs, platelet movement across the epithelium was proportional to the extent of PMN transmigration, and strategies that blocked PMN transmigration diminished platelet movement. Furthermore, platelet-PMN comigration was observed in intestinal tissue derived from human patients with inflammatory bowel disease (IBD). The translocated platelets were found to release large quantities of ATP, which was metabolized to adenosine via a 2-step enzymatic reaction mediated by ectonucleotidases, including CD73 and ecto-nucleoside triphosphate diphosphohydrolases (ecto-NTPDases), expressed on the apical membrane of the intestinal epithelial cells. In vitro studies and a mouse model of intestinal inflammation were employed to define a mechanism involving adenosine-mediated induction of electrogenic chloride secretion, with concomitant water movement into the intestinal lumen. These studies demonstrate that ecto-NTPDases are expressed on the apical membrane of epithelial cells and are involved in what we believe to be a previously unappreciated function for platelets in the inflamed intestine, which might promote bacterial clearance under inflammatory conditions.
\end{abstract}

\section{Introduction}

Several forms of active mucosal disease (e.g., inflammatory bowel disease [IBD]) feature a mixed inflammatory infiltrate predominantly consisting of polymorphonuclear leukocytes (PMNs) and monocytes (1). Accumulating evidence suggests that tissue injury in the mucosa is not the outcome of a dysregulated immune response exclusively but also involves the participation of other nonimmune cells such as platelets (2). Several lines of evidence support this hypothesis: First, platelet numbers are increased in IBD (2). Second, activated platelets secrete a broad variety of biologically active molecules, some of which are able to induce or amplify an inflammatory response of leukocytes. Third, platelets are known to play an essential role in leukocyte recruitment in the vasculature and hence trafficking into tissues (3). Moreover, it has been recently demonstrated in a model of experimental colitis that recruitment of leukocytes and that of platelets are codependent processes in inflamed colonic venules (4).

Nonstandard abbreviations used: Ado, adenosine; $\mathrm{AMLP}, N$-formyl-methionylleucyl-phenylalanine; IBD, inflammatory bowel disease; Isc, short circuit current; NPPB, 5-nitro-2-(3-phenylpropylamino)-benzoate; NTPDase, nucleoside triphosphate diphosphohydrolase; PMN, polymorphonuclear leukocyte; POM-1, polyoxometalate-1. Conflict of interest: The authors have declared that no conflict of interest exists. Citation for this article: J. Clin. Invest. 118:3682-3692 (2008). doi:10.1172/JCI35874.
It is now appreciated that during ongoing inflammation, infiltrating leukocytes can significantly influence tissue function through the liberation of soluble mediators and thus can promote the resolution of inflammation (5). One such pathway is adenine nucleotide metabolism. For example, a number of cell types can release ATP in an active manner during hypoxia and at sites of ongoing inflammation (6). The major pathway for extracellular hydrolysis of ATP and ADP is ecto-nucleoside triphosphate diphosphohydrolase (ecto-NTPDase-1) (7), previously identified as ecto-ATPase, ecto-ATPDase, or CD39 $(8,9)$. Its role to date has been to modulate platelet purinoreceptor activity by the sequential hydrolysis of extracellular ATP or ADP to $\operatorname{AMP}(9,10)$. Eight separate ENTPD genes encode members of the NTPDase protein family (11). AMP generated through this metabolic step can be further metabolized by ecto-5'-nucleotidase (CD73), a glycoprotein localized to the apical membrane surface of polarized epithelia (12). Surface-localized CD73 enzymatically converts adenine nucleotides (e.g., AMP) into adenosine (Ado), which in turn can activate transmembrane Ado receptors or can be internalized through dipyridamole-sensitive carriers (12).

Ado exerts paracrine and autocrine functions on most cell types. Pathophysiologic conditions of hypoxia/ischemia result in numerous adenine nucleotide metabolic changes, and Ado has a dem- 
A

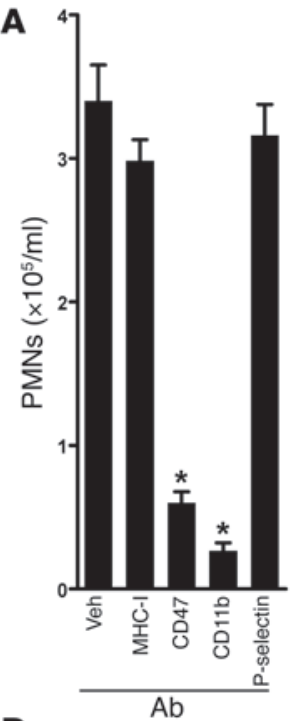

D

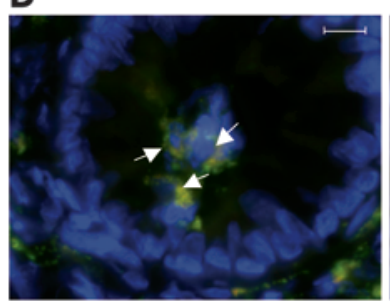

B
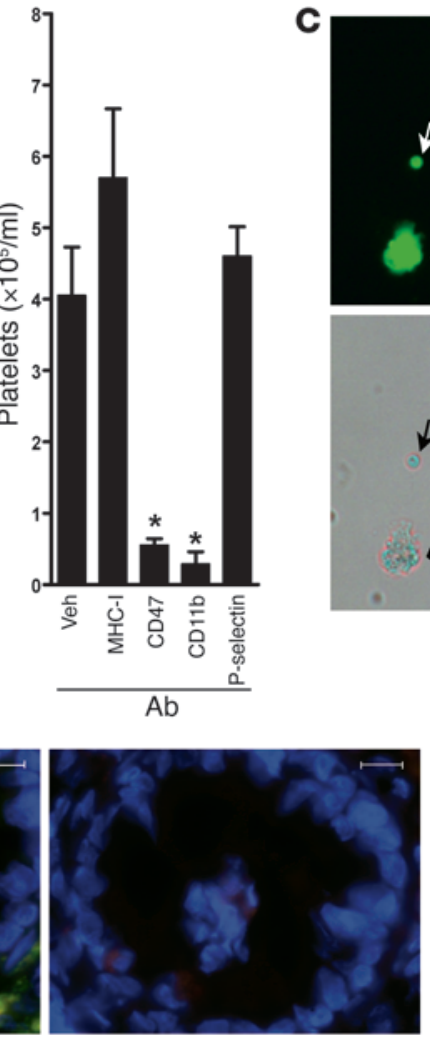
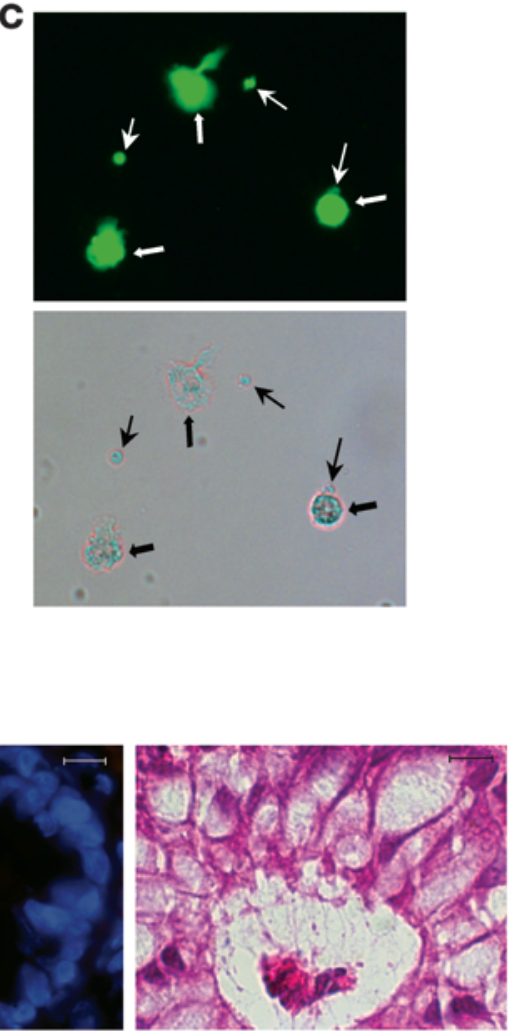

Figure 1

Influence of PMNs on platelet translocation. Activated PMNs $\left(10^{6} ; 10^{-6} \mathrm{M}\right.$ fMLP) were added together with $6 \times 10^{6}$ platelets to the basolateral surface of confluent T84 cells after preincubation with antibodies directed against CD11b, CD47, P-selectin, binding control (anti-MHC class I), or vehicle (Veh). Transmigrated PMNs and platelets were then assessed by microscopic evaluation (A and $\mathbf{B}$ ). Results are derived from 6 monolayers in each condition and are expressed as mean \pm SD. ${ }^{*} P<0.01$ compared with no-antibody control. (C) Transmigrated PMNs and platelets were incubated with $1 \mu \mathrm{g} / \mathrm{ml} \mathrm{BCECF}$ for 20 minutes at $37^{\circ} \mathrm{C}$. Cells were concentrated 4-fold in HBSS, and fluorescent cells were imaged by fluorescence microscopy. Shown here are examples of images after transmigration, where thick arrows indicate PMNs and thin arrows, platelets. Original magnification, $\times 400$. (D) Platelet-PMN cotransmigration in crypt abscesses from human IBD. Depicted here are merged fluorescence immunohistochemistry images showing localization of PMNs in green (anti-MPO), platelets in red (anti-CD41), and nuclei in blue (DAPI) (left panel); secondary antibody-only control (middle panel); and an adjacent section stained with H\&E demonstrating the crypt abscess (right panel). Arrows in the left panel indicate areas of platelet-PMN colocalization, shown in yellow. Scale bars: $10 \mu \mathrm{m}$.

onstrated role in organ function under such conditions. While the source of interstitial Ado in hypoxia/inflammation has been the basis of some debate, it is generally accepted that the dephosphorylation of AMP by CD73 represents the major pathway of Ado formation during oxygen supply imbalances (13). Presently, 4 subtypes of $\mathrm{G}$ protein-coupled Ado receptors have been identified, designated A1, A2A, A2B, and A3. These receptors are classified according to utilization of pertussis toxin-sensitive pathways (A1 and A3) or adenylate cyclase activation pathways (A2A and A2B) (14). Epithelial cells of many origins constitutively express Ado receptors, primarily of the A2A and A2B subtypes (15), wherein mucosal epithelial cells are one of the most enriched cell populations of A2BR (15).

Based largely on these studies, we hypothesized that platelets contribute to the luminal pathophysiology of mucosal inflammation. We therefore examined whether activated PMNs are able to influence platelet translocation during transmigration to sites of intestinal inflammation. Initial studies indicated that epithelial PMN transmigration appears concomitantly with the translocation of platelets. Since ion and fluid transport across epithelia are key features of intestinal epithelial cells, we investigated whether epithelial fluid homeostasis is altered in the presence of activated PMNs and platelets. Our results demonstrate that activation of both cell types induces chloride secretion on intestinal epithelial cells. Extensions of these findings identified ATP as a soluble secretagogue in the supernatants of PMNs and to an even higher degree in the supernatants of activated platelets. This platelet-PMN intestinal crosstalk pathway, identified here for the first time to our knowledge, likely serves as a robust defensive response by which mucosal surfaces are flushed from bacteria and bacterial products under inflammatory conditions.

\section{Results}

PMNs facilitate transepithelial platelet movement. PMNs are known to actively migrate across epithelial cells in a number of mucosal diseases (16). This event can be modeled in vitro by plating epithelial cells on the underside of permeable membrane supports and stimulating isolated PMN movement through the use of a transepithelial chemotactic gradient (17). It is not currently known whether other cells found in mixed inflammatory infiltrates, such as platelets, might also move across epithelial monolayers. Therefore, we sought to examine whether platelets transmigrate. Initial experiments indicated that while PMNs readily transmigrate across epithelia, studies with purified platelets in the presence or absence of collagen stimulation revealed no significant increase in platelet movement across T84 intestinal epithelial monolayers (data not shown).

Extensions of these studies using mixed populations of PMNs and platelets, however, revealed that platelets appear to "follow" PMNs across epithelial monolayers. Indeed, as shown in Figure 1, A-C, platelet movement across the epithelium correlated with PMN migration. Blockade of PMN transmigration with functionally inhibitory antibodies directed against CD47 (clone C5D5) (18) resulted in a proportional decrease in platelet movement (Figure 1, A-C) across intestinal epithelial cells $(81 \% \pm 5 \%$ and $85 \% \pm 7 \%$ decrease in PMN and platelet translocation, respectively; $P<0.01$ compared with noantibody control). Similarly, inhibition of PMN transmigration with antibodies directed against CD11b (clone 44a) (19) revealed a proportional decrease in platelet movement $(87 \% \pm 6 \%$ and $89 \% \pm 6 \%$ decrease in PMN and platelet translocation, respectively; $P<0.01$ compared with no-antibody control). Functionally inhibitory antibodies directed against P-selectin did not influence either PMN or 

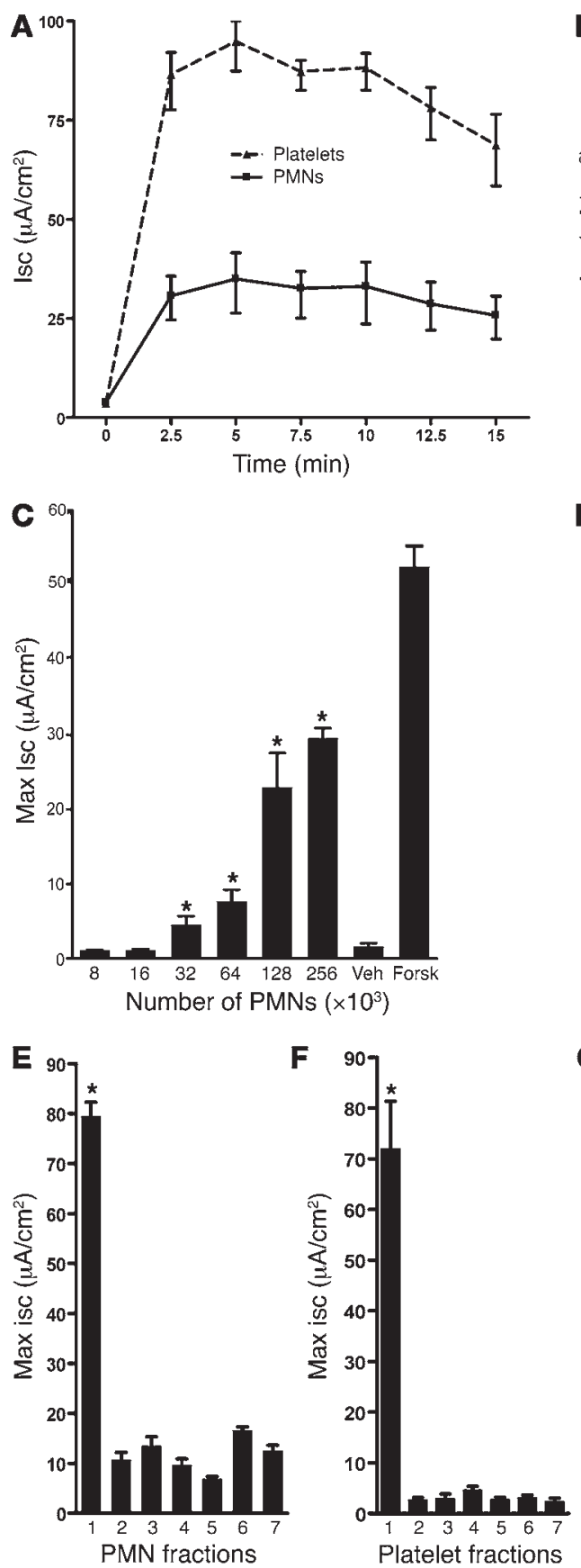

Platelet fractions
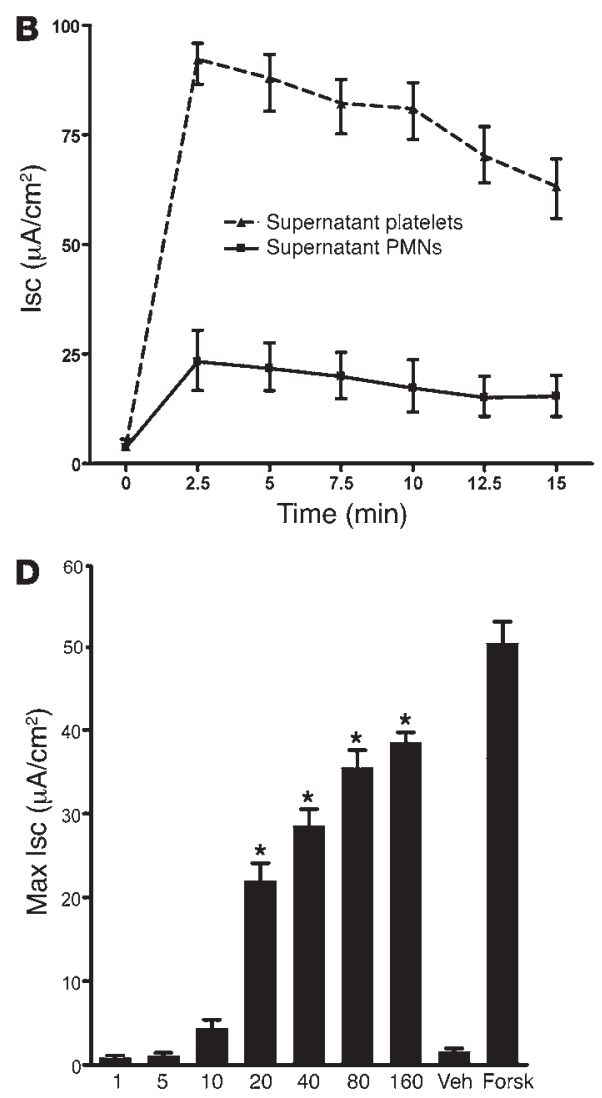

Number of plateletss $\left(\times 10^{3}\right)$

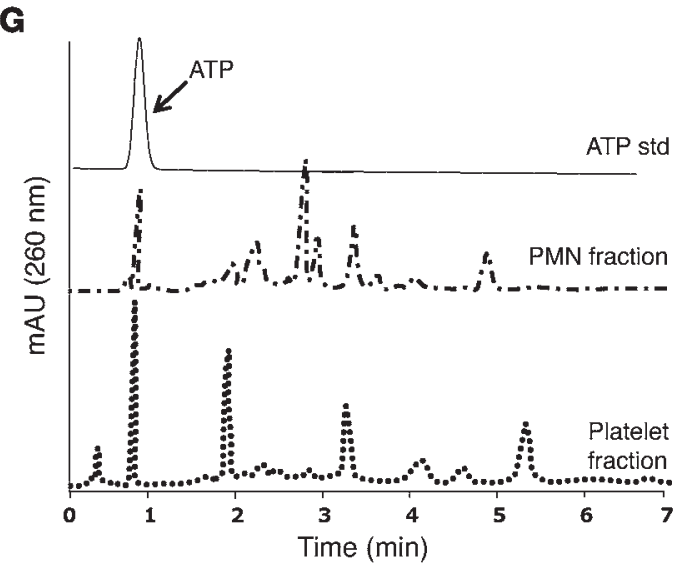

Figure 2

Characterization of T84 cell monolayer response to activated $\mathrm{PMNs}$ and platelets. (A and B) Activated cells and their supernatants were applied to the apical side. (C and $\mathbf{D}$ ) Dose response with increasing numbers of PMNs and platelets. ${ }^{*} P<0.01$ compared with vehicle. Max, maximum; Forsk, forskolin. (E and F) Supernatants from activated PMNs $\left(10^{-6} \mathrm{M}\right.$ fMLP for 10 minutes) and activated platelets were fractionated by HPLC. Samples were concentrated and tested for bioactivity on T84 monolayers. ${ }^{*} P<0.001$, fraction 1 compared with other fractions. Data represent mean \pm SD from 3 separate experiments. (G) Chromatographic identification of ATP in supernatant derived from platelets and PMNs. A representative overlay chromatogram of activated PMN and platelet supernatant and native ATP with 1 dominant peak at $260 \mathrm{~nm}$. ATP std, ATP standard platelet translocation compared with either no antibody or binding control antibodies (Figure 1, A and B, respectively; $P=\mathrm{NS}$ ). All PMN transmigration results were confirmed using a myeloperoxidase enzyme assay $(70 \% \pm 5 \%$ and $75 \% \pm 6 \%$ decrease in PMN translocation with anti-CD11b and anti-CD47, respectively; $P<0.05$ compared with no-antibody control; data not shown). Binding control antibodies directed against MHC class I did not significantly influence either PMN or platelet movement across epithelial monolayers $(P=$ NS comparing no-antibody controls with anti-MHC class I for both PMN and platelet quantification).

To document the pattern of translocated PMNs and platelets, transmigrated cells were gently harvested, loaded with BCECF, and observed by fluorescence microscopy. Figure 1C shows a representative image of BCECF-loaded PMNs and platelets following transepithelial migration. Both individual platelets and plateletPMN complexes were observed under these circumstances. To demonstrate in vivo relevance for such observations, we examined whether platelet-PMN complexes exist during inflammation in a classic model of PMN transepithelial migration; namely, crypt abscesses associated with human IBD. To do this, we obtained colonic biopsy samples from patients with a definitive diagnosis of IBD with crypt abscesses and used immunofluorescence histochemistry for localization of platelets and PMNs within crypt abscesses. For these purposes, we used antibodies directed against 

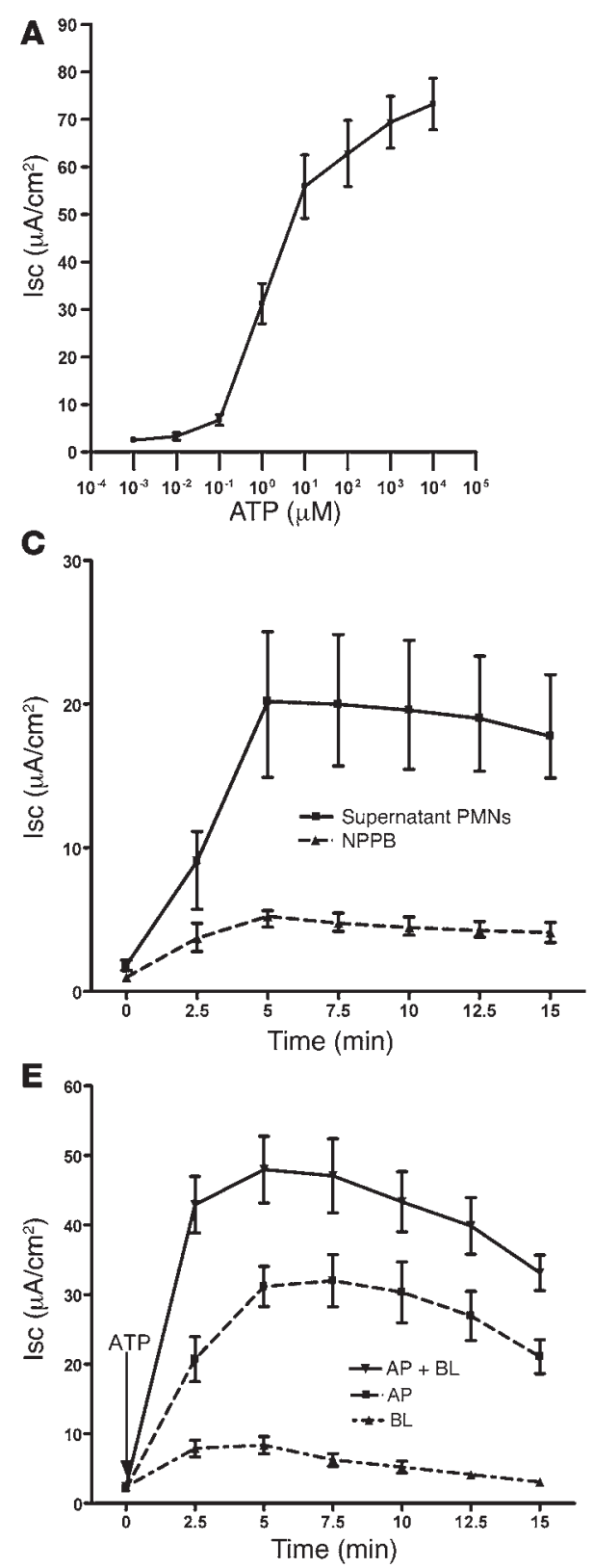
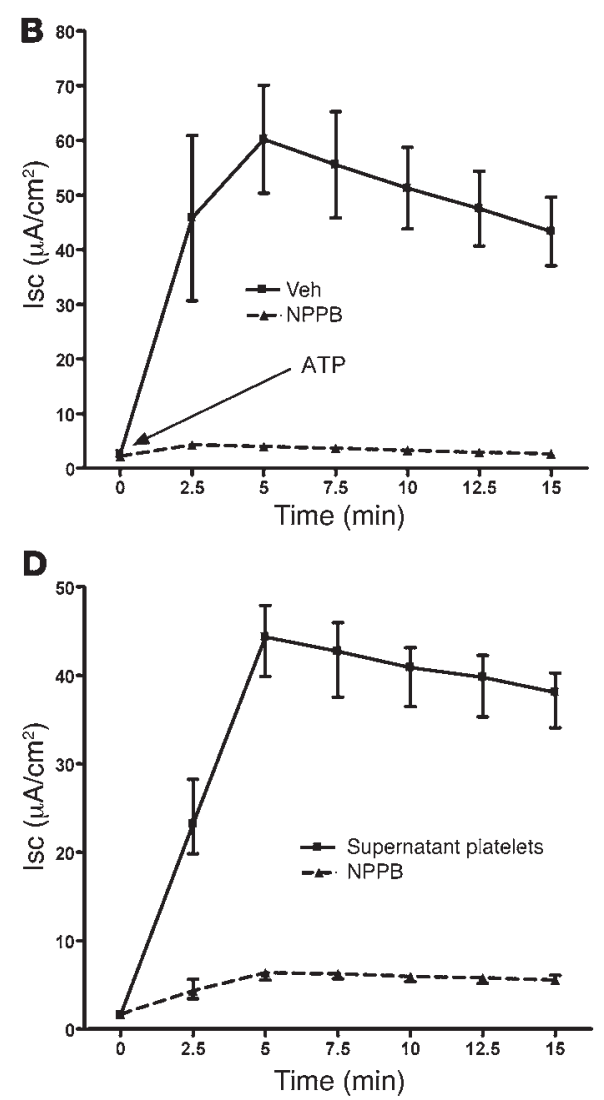

Figure 3

Contribution of epithelial chloride channels to the $\mathrm{Cl}^{-}$secretion response. (A) Epithelial electrogenic $\mathrm{Cl}^{-}$secretion in response to increasing concentrations of ATP. Results are pooled from 8 monolayers in each condition and expressed as mean \pm SEM. (B) Influence of chloride channel inhibitor NPPB on Isc response elicited by ATP. Results are pooled from 6 monolayers in each condition and results are expressed as mean \pm SEM. (C and $\mathbf{D}$ ) Influence of NPPB on electrogenic $\mathrm{Cl}^{-}$secretion elicited by supernatants from activated PMNs (C) or activated platelets (D). Results are pooled from 9 monolayers and supernatants derived from 3 separate donors in each condition, and results are expressed as mean \pm SEM. (E) Polarity of epithelial electrogenic $\mathrm{Cl}^{-}$secretion in response to $10 \mu \mathrm{M}$ ATP to the apical (AP), basolateral $(\mathrm{BL})$, or apical and basolateral (AP $+\mathrm{BL})$ surfaces of confluent T84 cell monolayers. Data are pooled from 6 monolayers in each condition, and results are expressed as mean \pm SEM.
GPIIb/IIIa (CD41; platelets) and myeloperoxidase (neutrophils). As shown in Figure 1D, this strategy revealed that PMNs localized within the lumen are studded with platelets following translocation across the epithelium in human IBD. Similarly, such findings were evident in more than $30 \%$ of crypt abscesses observed (multiple crypt abscesses from $n=5$ patients), suggesting that this association is not a minor event during inflammation. These observations in vitro and ex vivo indicate that PMN transmigration facilitates platelet translocation across epithelial monolayers.

Influence of platelets on epithelial function. We extended these findings to determine whether apically localized platelets (i.e., translocated) influence epithelial functional responses. We concentrated on 2 primary epithelial endpoints, namely, barrier function and ion transport. Initial studies revealed that, as measured as transepithelial resistance, activated platelets do not significantly influence epithelial barrier function (data not shown). However, examina- tion of ion transport responses revealed a quite different scenario. Specifically, thrombin-activated platelets elicit a prominent induction of electrogenic chloride secretion, measured as an increase in short circuit current (Isc). As shown in Figure 2A, the addition of platelets (final concentration, $1 \times 10^{7}$ platelets $/ \mathrm{ml}$ ) to the apical surface of T84 monolayers in the presence of collagen $(1 \mu \mathrm{g} / \mathrm{ml})$ elicited a rapid and sustained increase in Isc $(P<0.001$ by ANOVA). Similarly, and as shown previously (20), $N$-formyl-methionylleucyl-phenylalanine-activated (fMLP-activated; $100 \mathrm{nM}$ ) PMNs (final concentration, $1 \times 10^{7} \mathrm{PMN} / \mathrm{ml}$ ) stimulated a prominent increase in Isc $(P<0.001$ by ANOVA). Additionally, we examined whether antibody-mediated inhibition of platelet P-selectin might influence chloride $\left(\mathrm{Cl}^{-}\right)$secretion in the presence of neutrophils. To do this, purified platelets and purified neutrophils were incubated with anti-P-selectin, thrombin activated, and examined for induction of electrogenic $\mathrm{Cl}^{-}$secretion. The results indicated that 

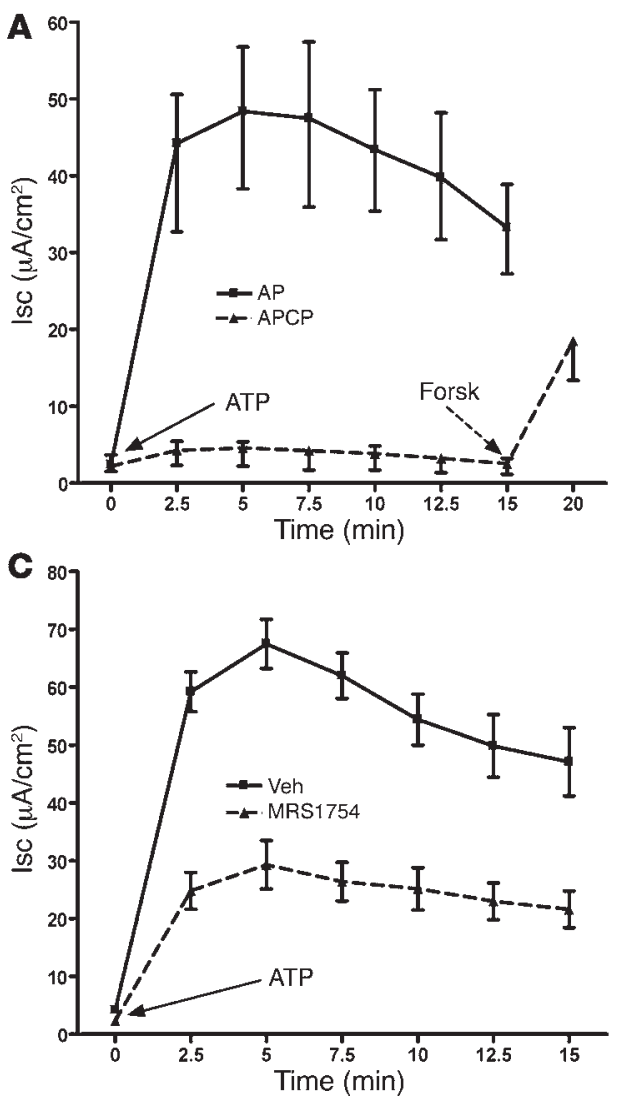
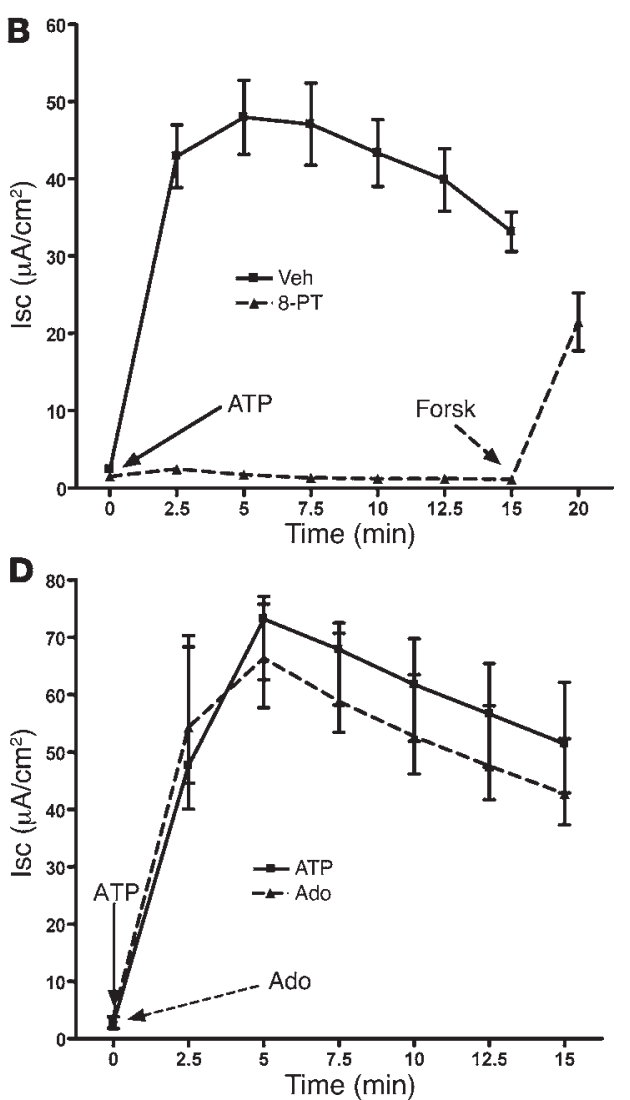

\section{Figure 4}

Characterization of relative contributions of epithelial apical membrane ecto-5'-nucleotidase (CD73) and Ado receptors on the $\mathrm{Cl}^{-}$secretion response elicited by ATP and Ado. (A) Effect of ecto-5'-nucleotidase inhibitor $\alpha$ - $\beta$-methylene ADP on Isc response elicited by ATP. T84 monolayers were preincubated with $20 \mu \mathrm{M} \alpha$ - $\beta$-methylene ADP for 5 minutes. (B) Effect of Ado receptor inhibitor 8-phenyltheophylline (8-PT) on Isc response elicited by ATP. T84 monolayers were preincubated with $20 \mu \mathrm{M}$ $\alpha$ - $\beta$-methylene ADP for 5 minutes. (C) Influence of Ado A2B receptor (AdoRA ${ }_{2 b}$ ) antagonist MRS1754 (10 $\mu \mathrm{M})$ on Isc response elicited by ATP. T84 monolayers were preincubated with $10 \mu \mathrm{M}$ MRS1754 for 5 minutes. (D) Time course of authentic ATP and Ado (both $10 \mu \mathrm{M}$ ) on the Isc response. All data are pooled from 6 monolayers in each condition, and results are expressed as mean \pm SEM. inhibition of P-selectin did not significantly influence $\mathrm{Cl}^{-}$secretion in this model (maximal Isc, $58 \pm 6$ and $51 \pm 7 \mu \mathrm{A} / \mathrm{cm}^{2}$ in the presence and absence of anti-P-selectin, respectively; $P=\mathrm{NS}$ ).

Extensions of these studies revealed that it is not likely that this response is specific for platelets and PMNs, since other leukocyte populations, to a lesser extent, also elicit electrogenic $\mathrm{Cl}^{-}$secretion. For example, the addition of purified fMLP-activated mononuclear cells (mixed monocytes and lymphocytes; $1 \times 10^{7} / \mathrm{ml}$ ) to intact epithelia resulted in a significant increase in $\mathrm{Cl}^{-}$secretion (Isc of $\left.6 \pm 0.2 \mu \mathrm{A} / \mathrm{cm}^{2} ; P<0.05\right)$, but far less than that observed with activated PMNs (Isc of $26 \pm 4.1 \mu \mathrm{A} / \mathrm{cm}^{2} ; P<0.001$ ) or activated platelets (Isc of $42 \pm 5.2 \mu \mathrm{A} / \mathrm{cm}^{2} ; P<0.001$ ). Thus, it is likely that all leukocyte populations, to some extent, elicit electrogenic $\mathrm{Cl}^{-}$secretion when incubated with epithelia.

To investigate the mechanism underlying this activity, we examined the influence of acellular, activated PMN or platelet supernatants on epithelial Isc responses. Briefly, human PMNs and platelets were isolated and suspended at a concentration of $10^{7} / 150 \mu \mathrm{l}$ buffer (a PMN concentration equivalent to $10^{7} \mathrm{PMNs}$ or platelets per insert) and stimulated with fMLP $\left(10^{-6} \mathrm{M}\right)$ or collagen $(1 \mu \mathrm{g} / \mathrm{ml})$ with constant agitation. After 60 minutes, this suspension was centrifuged ( $400 \mathrm{~g}, 10$ minutes). The supernatant was removed, passed through a $0.2-\mu \mathrm{m}$ filter, and adjusted to a $\mathrm{pH}$ of 7.4. Supernatant was then added to T84 cell inserts and monitored for changes in Isc. The results are shown in Figure 2B. Acellular supernatants derived from both platelets and PMNs resulted in a prominent induction of Isc (both $P<0.001$ compared with vehicle controls). Like our findings with intact cells, platelet supernatants elicited a larger increase in Isc compared with PMN supernatants
$(P<0.01$ by ANOVA). To rule out that these differences between PMNs and platelets did not reflect a saturation in either cell number or Isc response, we compared Isc responses from supernatants derived from various numbers of activated PMNs and activated platelets. As shown in Figure 2, C and D, supernatants derived from both PMNs and platelets elicited a concentration-dependent increase in Isc $(P<0.01$ for both by ANOVA). Vehicle and forskolin controls are also shown for comparison. A plot of these results and linear regression of these plots revealed that on a per-cell basis, platelet supernatants elicited a $3.2 \pm 0.4$-fold increase in Isc compared with PMN supernatants $(P<0.01)$.

Since activated PMNs can elicit a strong oxidative stress response, we examined whether oxidizing conditions might influence electrogenic $\mathrm{Cl}^{-}$secretion during platelet-PMN interactions. To do this, purified platelets and purified neutrophils were incubated with the reducing agent DTT $(100 \mu \mathrm{M})$, fMLP activated, and examined for induction of electrogenic $\mathrm{Cl}^{-}$secretion. These studies showed that DTT diminished $\mathrm{Cl}^{-}$secretion elicited by PMN activation in the presence of platelets (maximal Isc, $49 \pm 6$ and $31 \pm 4 \mu \mathrm{A} / \mathrm{cm}^{2}$ in the presence and absence of DTT, respectively; $P<0.05$ ). Such results indicate the likelihood that oxidative stress during platelet-PMN interactions contribute to the liberation. Taken together, such findings suggest that both activated platelets and activated PMNs secrete factor(s) that elicit epithelial electrogenic chloride secretion as measured by Isc.

Identification of secreted ATP as a mediator of epithelial $\mathrm{Cl}^{-}$secretion. We next sought to define the nature of the soluble mediators derived from activated PMNs and platelets that contribute to the stimulation of Isc shown in Figure 2. To do this, we used HPLC to fraction- 

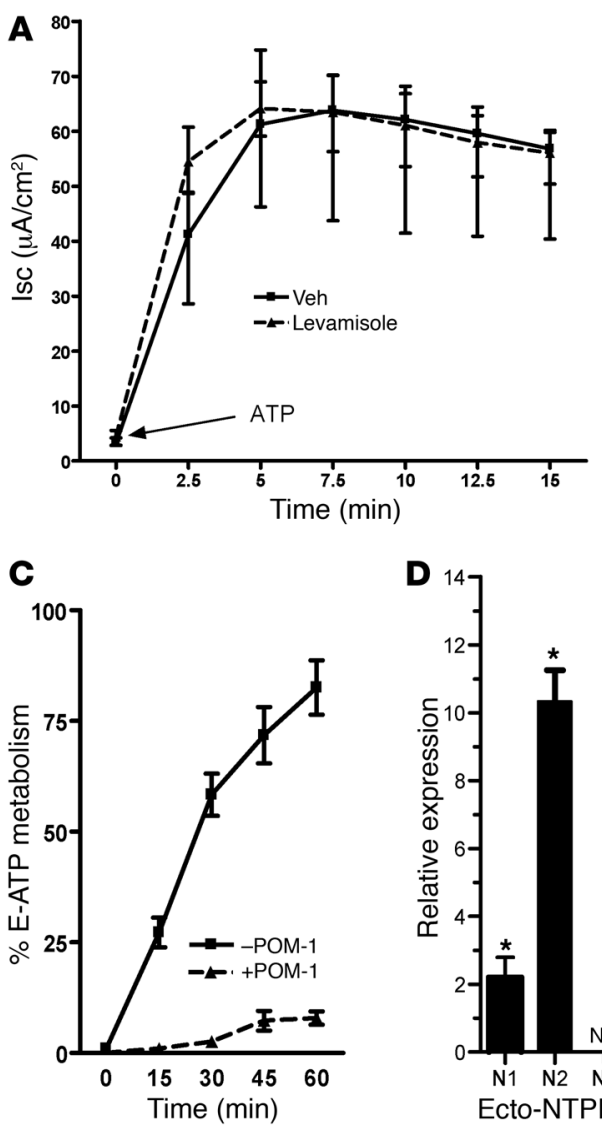

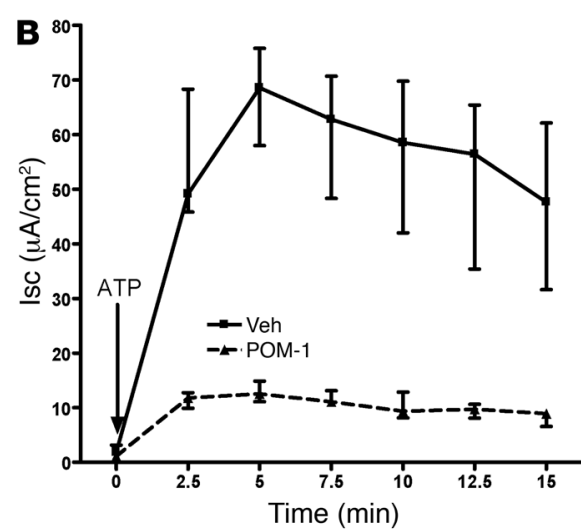

E

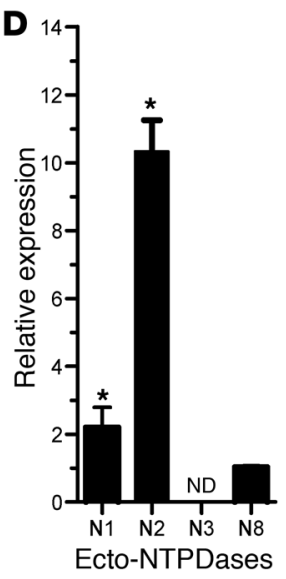

\section{Figure 5}

Role of ecto-NTPDases in ATP-elicited changes in $\mathrm{Cl}^{-}$secretion response within intestinal epithelial cells (IECs). (A) Characterization of relative contributions of epithelial apical alkaline phosphatase to the Isc response elicited by ATP. T84 monolayers were preincubated with $10 \mu \mathrm{M}$ levamisole for 5 minutes. (B) Inhibition of ecto-NTPDases by POM-1 $\left(\mathrm{Na}_{6}\left[\mathrm{H}_{2} \mathrm{~W}_{12} \mathrm{O}_{40}\right]\right)(10 \mu \mathrm{M})$ abolishes the effects of ATP-elicited changes on Isc. (C) Functional decrease of NTPDase surface activity by POM-1. T84 monolayers were washed, and NTPDase activity was determined by HPLC analysis of E-ATP conversion to E-AMP in the presence and absence of POM-1. (D and E) Expression of ecto-NTPDases on IECs. Total RNA was isolated from confluent T84 monolayers using real-time (D) and conventional PCR (E). As shown, $\beta$-actin transcript was determined in parallel and used as a control. ${ }^{\star} P<0.01$ compared with NTPDase-8 (N8). Data are derived from 3 monolayers in each condition and expressed as mean \pm SEM. $\mathrm{ND}$, not detected. ate supernatants derived from activated PMNs and from activated platelets and tested bioactivity of individual fractions on epithelia. As shown in Figure 2, E and F, of 7 fractions that were isolated, epithelial Isc was only significantly increased in fraction 1 compared with buffer controls. Parallel spectral scans from eluted samples revealed a dominant UV peak at $260 \mathrm{~nm}$ for fraction 1 (data not shown), a spectral profile consistent with nucleotides (21).

In the past, we have reported that PMNs release AMP during transmigration $(21,22)$. More recently, we have demonstrated that PMNs release ATP in an activation-dependent manner (23). Based on these findings, and the fact that platelets are a known source of extracellular ATP, we compared retention times of native ATP with the retention times of the HPLC fractions from activated platelets and activated PMNs. The retention time of ATP was identical to that of both platelet and PMN fraction 1, and coinjection with native ATP revealed a single peak, thereby identifying fraction 1 as ATP (Figure $2 \mathrm{G}$ ). To verify ATP release from activated platelets, we used standard luminometric ATP detection assays. ATP rapidly accumulated in supernatants derived from collagen-activated platelets (maximal level, $16.8 \pm 2.1 \mathrm{nmol} / 10^{7}$ platelets after 5 -minute activation). These results indicate that both activated platelets and activated PMNs rapidly release ATP and that such extracellular ATP promotes electrogenic $\mathrm{Cl}^{-}$secretion in intestinal epithelia.

Native ATP induces epithelial $\mathrm{Cl}^{-}$secretion. Having demonstrated that activated platelets and PMNs release ATP, we next examined the influence of the authentic ATP on epithelial ion transport. As shown in Figure 3A, the addition of native ATP (concentration range, $1 \mathrm{nM}-10 \mathrm{mM}$ ) induced a concentration-dependent increase in Isc $(P<0.001$ by ANOVA). This response to ATP was inhibited with the
CFTR inhibitor 5-nitro-2-(3-phenylpropylamino)-benzoate (NPPB) (Figure 3B), indicating that this Isc response reflects electrogenic $\mathrm{Cl}^{-}$secretion. Moreover, NPPB strongly inhibited electrogenic $\mathrm{Cl}^{-}$ secretion induced by both platelets and neutrophils. Indeed, NPPB blocked electrogenic $\mathrm{Cl}^{-}$secretion by $87 \% \pm 5 \%$ and $81 \% \pm 7 \%$ (both $P<0.001)$ for platelet and neutrophil-elicited responses, respectively, while NPPB blocked ATP-elicited electrogenic $\mathrm{Cl}^{-}$secretion by $91 \% \pm 8 \%$. Such findings strongly implicate CFTR-dependent $\mathrm{Cl}^{-}$ secretion as the mechanism underlying this response.

ATP-stimulated electrogenic $\mathrm{Cl}^{-}$secretion was highly polarized toward the apical plasma membrane. As shown in Figure 3E, monitoring Isc following the addition of equimolar ATP to apical, basolateral, or apical and basolateral surfaces resulted in a greater than 3 -fold predominant response from the apical aspect of epithelial monolayers. Such findings suggest that ATP induces electrogenic $\mathrm{Cl}^{-}$secretion predominantly from the apical membrane surface.

To gain insight into potential mechanisms of ATP regulation of electrogenic $\mathrm{Cl}^{-}$secretion, we reasoned that ATP may directly activate surface ATP receptors or, alternatively, may be metabolized to other nucleotides/nucleosides at the membrane surface. Based on these findings, we determined whether ATP metabolism is important in the induction of epithelial electrogenic $\mathrm{Cl}^{-}$secretion. First, intestinal epithelial cells are known to express ecto-5'nucleotidase (CD73) at high levels on the apical membrane (24, $25)$. This surface enzyme converts AMP to Ado and functions as the terminal step in the formation of extracellular Ado (26). Thus, we examined the influence of the CD73 antagonist APCP on ATP-stimulated electrogenic $\mathrm{Cl}^{-}$secretion. As shown in Figure 4A, APCP inhibited maximal Isc responses induced by ATP 

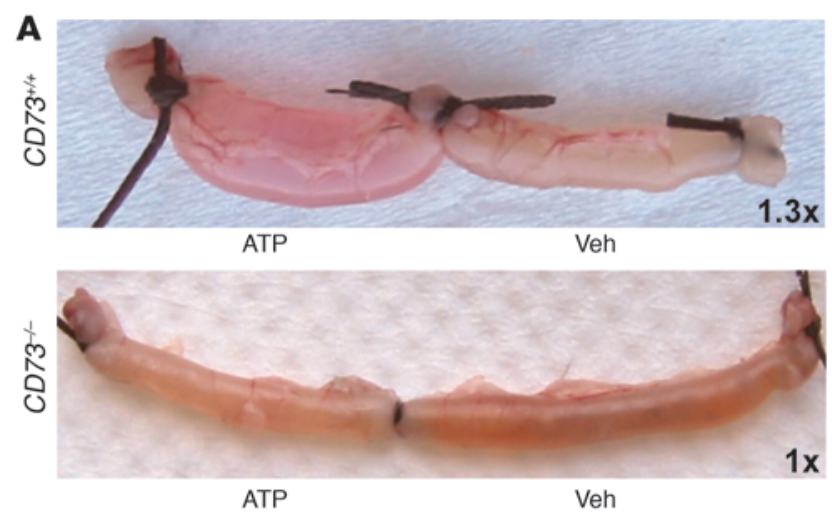

B

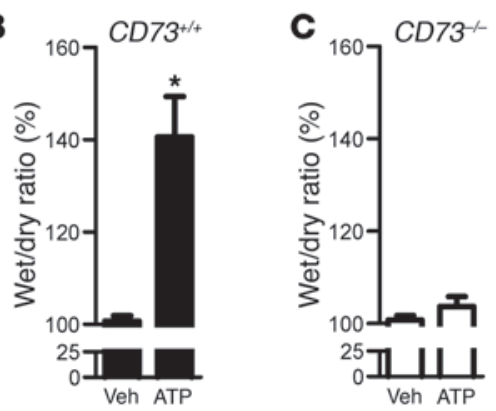

$(10 \mu \mathrm{M})$ by $94 \% \pm 6 \%(P<0.001)$. Such responses did not reflect the inability of cells to secrete $\mathrm{Cl}^{-}$, as evidenced by a rapid Isc response following the addition of forskolin (Figure 4A). Second, we determined the relative influence of Ado in this response by using the nonspecific Ado receptor antagonist 8-phenyltheophylline (8-PT). As shown in Figure 4B, electrogenic $\mathrm{Cl}^{-}$secretion associated with the addition of native ATP $(10 \mu \mathrm{M})$ on epithelial monolayers was completely blocked by 8 -PT $(10 \mu \mathrm{M} ; P=$ NS compared with buffer alone), indicating that the Isc response observed with ATP reflects Ado receptor activation. Third, we examined whether more specific Ado receptor inhibitors would block this response. As shown the Figure 4C, the selective Ado A2B receptor antagonist MRS1754 significantly attenuated ATP-stimulated Isc $(P<0.01)$. Finally, we compared Isc responses to equimolar concentrations of ATP and Ado. As shown in Figure 4D, both Ado and ATP elicited rapid and sustained electrogenic $\mathrm{Cl}^{-}$secretion, with no significant differences observed over the time course examined ( $P=$ NS by ANOVA). Such findings implicate the apical metabolism of ATP to Ado as critical to electrogenic $\mathrm{Cl}^{-}$secretion elicited by ATP.

Expression of ecto-NTPDase activity on intestinal epithelia. Based on the findings above, we sought to define the presence of surface membrane activity responsible for ATP conversion to AMP (i.e., CD73 substrates; see Figure 4A). A number of enzymes can metabolize ATP to AMP, including alkaline phosphatase and ecto-NTPDases (27). Given that intestinal epithelia express high levels of alkaline phosphatase (28), we determined whether the alkaline phosphatase inhibitor levamisole would influence ATP-stimulated Isc. As shown in Figure 5A, levamisole did not influence ATP-stimulated $\mathrm{Cl}^{-}$secretion $(P=\mathrm{NS})$, suggesting that it is unlikely that alkaline phosphatase significantly contributes to extracellular ATP metabolism.

To date, the expression of ecto-NTPDases on intestinal epithelial cells have not been reported. Ecto-NTPDases are a family of surface enzymes with important documented roles in thromboregulation

\section{Figure 6}

Role of ATP-elicited changes in chloride secretion in vivo. $C D 73^{-/-}$mice and age-, weight-, and sex-matched controls were administered $1 \mathrm{mM}$ ATP solution into sutured loops of the small intestine and vehicle solution into adjacent loops. Animals were kept under anesthesia the whole time and killed 1 hour after ATP injection. Intestinal loops were taken out and compared (A). Furthermore, wet/dry ratios were performed separately for each intestinal loop (B and $\mathbf{C})$. Data are presented as mean \pm SEM wet/dry ratio and are derived from $n=7$ knockout mice and $n=7$ control animals. ${ }^{*} P<0.01$, vehicle injection compared with ATP injection.

and hemostasis (27). To examine the relative contribution of ectoNTPDases to ATP-stimulated electrogenic $\mathrm{Cl}^{-}$secretion, the specific ecto-NTPDase antagonist polyoxometalate-1 (POM-1) (29) was used. As shown in Figure 5B, POM-1 $(10 \mu \mathrm{M})$ inhibited ATPstimulated $(10 \mu \mathrm{M})$ Isc by $86 \% \pm 8 \%(P<0.01)$, thereby implicating ecto-NTPDase activity in ATP-stimulated electrogenic $\mathrm{Cl}^{-}$secretion. Further analysis using HPLC-based analysis of etheno-ATP conversion to etheno-AMP (30) revealed prominent ecto-NTPDase activity on the surface of T84 cells $(64 \% \pm 8 \%$ conversion of 100 $\mu \mathrm{M}$ ATP in 60 minutes; Figure 5C). Similar to the Isc response, this activity was inhibited by nearly $90 \%$ in the presence of POM-1 $(7 \% \pm 3 \%$ conversion of $100 \mu \mathrm{M}$ in 60 minutes; Figure $5 \mathrm{C}$; $P<0.001)$.

Based on the functional expression of ecto-NTPDases, we screened T84 cell mRNA for the existence of various members of the ecto-NTPDase family. As shown in Figure 5D (real-time PCR) and Figure 5E (conventional PCR), T84 intestinal epithelial cells expressed at least 3 members of the ecto-NTPDase family, with rank order of expression NTPDase-2 >>NTPDase-1>NTPDase-8. The other surface NTPDase (NTPDase-3) was not detectable by either method used. Unfortunately, reagents available for protein analysis (e.g., antibodies, ELISAs) are not readily available, and therefore, we were not able to quantify protein expression.

Expression of ecto-NTPDase activity on intestinal epithelia. As proof of principle for ecto-NTPDase function on intestinal epithelia, we extended these in vitro observations to an in vivo model. As shown in Figure 6A, we used a murine colonic loop ligation model to compare fluid transport elicited by luminal application of buffer or native ATP ( $1 \mathrm{mM}$ final concentration). To directly compare responses and to minimize colon-colon variability, adjacent sections of colon were administered either ATP or vehicle (Figure 6A). As shown in Figure 6B, over a 60-minute time frame, ATP elicited a large increase in fluid movement to the luminal aspect relative to vehicle $(41 \% \pm 7 \%$ increase in wet/dry ratio; $n=7)(P<0.01)$.

To demonstrate specificity for nucleotidase activity in this model, we used $C D 73^{-/-}$mice. A comparison of ATP-stimulated fluid movement and examination of wet/dry ratios between $C D 73^{+/+}$and $\mathrm{CD} 73^{-/}$animals revealed prominent differences between colons derived from these mouse strains (Figure 6). Specifically, ATP did not significantly stimulate fluid movement, reflected as no change in the wet/dry ratio (Figure 6C; $P=$ NS compared with vehicle, $n=7$ ). Such observations strongly implicate ecto-nucleotidase activity as a mechanism central to ATP-stimulated fluid transport. Moreover, since ATP is not a substrate for CD73, these studies identify epithelial ecto-NTPDase as a previously unappreciated mechanism for ATP-stimulated luminal fluid transport.

\section{Discussion}

Mucosal diseases of tissues lined by an epithelium are often characterized by a mixed inflammatory infiltrate, including PMNs, 


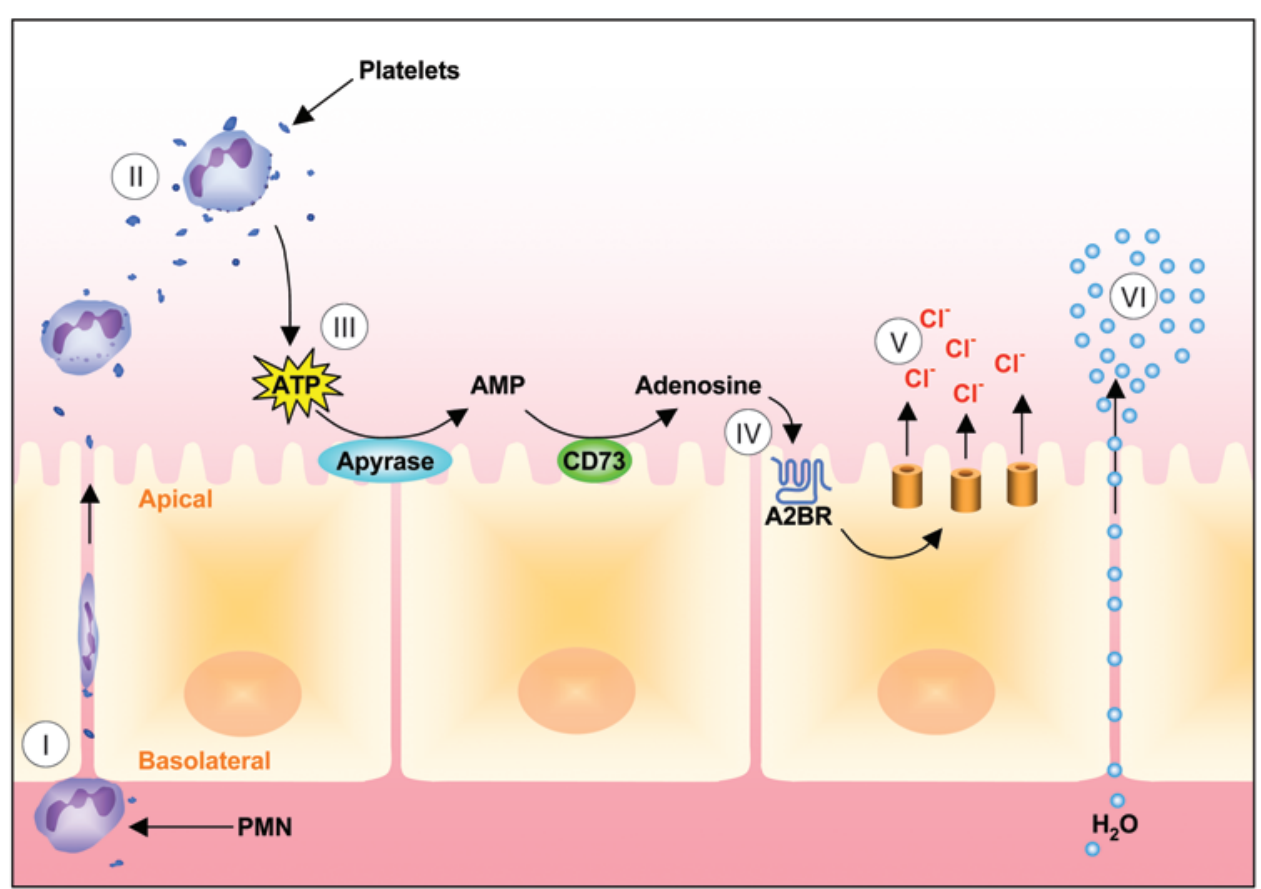

Figure 7

Proposed model of facilitated platelet translocation and activation of epithelial electrogenic $\mathrm{Cl}^{-}$secretion during PMN transmigration. During active inflammation, platelets are caught in the flow of PMN transmigration, resulting in platelet translocation across the apical side of mucosal epithelial cells (I). PMNand platelet-derived ATP (II) is selectively metabolized to Ado by a 2-step enzymatic reaction involving ecto-apyrase and ecto-nucleotidase (CD73) (III). Ado binding to apical Ado A2B receptors (A2BR; IV) results in activation of electrogenic $\mathrm{Cl}^{-}$secretion $(\mathrm{V})$ and the paracellular movement of water (VI). Such a platelet/PMN-epithelial crosstalk pathway may serve as a defensive response by which mucosal surfaces are flushed from bacteria and bacterial products under inflammatory conditions.

monocytes, lymphocytes, and platelets. Likewise, a number of studies have suggested that platelet interactions with leukocytes amplify a number of inflammatory features (3). In this study, we examined the translocation of platelets across mucosal epithelial cells. Initial studies revealed that platelets, per se, lack the ability for significant migration capacity. However, in the presence of PMNs, platelets appear to "follow" PMNs across the epithelium. Extensions of these studies revealed functional outcomes of platelets in the lumen; namely, that activated platelets release large quantities of ATP, which is subsequently metabolized to Ado via a 2-step enzymatic reaction. Ado liberated in this manner is made available to receptors to activate electrogenic $\mathrm{Cl}^{-}$secretion in vitro and in vivo (Figure 7). These studies reveal a previously unappreciated role for platelets in the mucosal lumen and identify the expression of ecto-NTPDase on the apical epithelial membrane.

Platelets are widely accepted as participants in the inflammatory response. While the thromboregulatory role for platelets is well established through decades of solid work, their role in regulating leukocyte recruitment and function has been appreciated more recently (3). Original studies by Marcus et al. $(31,32)$ revealed that platelet-PMN interactions defined a new biochemical pathway, termed transcellular biosynthesis, whereby platelets and PMNs specifically interact to liberate lipid intermediates that form the basis for leukotriene production. Subsequent studies revealed that such platelet-PMN transcellular biosynthesis can liberate non-leukotriene lipoxygenase interaction products, termed lipoxins $(33,34)$. More recently, it has been appreciated that platelet-PMN interactions amplify inflammatory responses through a number of mechanisms (3). Specifically, platelets interact with PMNs through at least 5 separate receptor-ligand pairs, including (platelet-PMN) CD40-CD40, GPIIb/IIIa-Mac-1, GPIIba-Mac-1, ICAM-2-LFA-1, and P-selectinP-selectin glycoprotein ligand-1 (P-selectin-PSGL-1) (3). While we do not know the exact molecular details involved in platelet movement across the epithelium, for a number of reasons it is likely to be complicated and involve multiple pathways. First, platelets do not appear to migrate across the epithelium in our model. This is not surprising, given that little evidence exists that platelets have the machinery for significant directed chemotaxis. Second, staining of comigrated cell types revealed both PMN-associated and non-PMNassociated platelets. This observation suggests that platelets may be carried into the lumen attached to PMNs (i.e., "piggyback" on PMNs). Likewise, it is possible that platelets could be caught in the streaming of PMNs through the epithelial paracellular space but not adherent to the PMNs. Third, the use of functionally inhibitory antibodies directed against a PMN ligand (CD11b/18) and an epithelial ligand (CD47) was revealing. Indeed, blockade of either ligand independently resulted in a proportional decrease in platelet accumulation on the luminal side of the epithelium. These observations support the idea that platelets follow PMNs across the epithelium in a concerted manner.

The role of nucleotides/nucleosides in inflammatory diseases is currently an area of intense investigation. Here, we demonstrate that both platelets and PMNs release large quantities of ATP at the luminal aspect of epithelia. While PMNs and platelets are established sources for extracellular nucleotide substrates (3), it is likely that all inflammatory cells release adenine nucleotides in the form of ATP or ADP. Indeed, multiple pathways exist to release ATP into the extracellular milieu, including exocytosis of ATP-containing vesicles, transport via connexin hemichannels, and direct transport through $\mathrm{ABC}$ proteins (35). Platelets have well-established dense granule degranulation mechanisms for ATP release (3), while PMNs and other cell types utilize connexin 43-mediated ATP release (36). In this regard, other inflammatory cells including lymphocytes and macrophages also express connexin $43(37,38)$ and thus likely have the capacity to liberate ATP in this fashion. Moreover, since ATP release by PMNs (and presumably other leukocytes) is activation dependent (23) and platelets enhance leukocyte activation through mechanisms involving surface P-selectin-PSGL-1 (3), it is probable that platelets may 
enhance leukocyte ATP release. Further studies will be necessary to define these principles.

Consistent with a number of previous studies $(26,39)$, the present results highlight extracellular nucleotide phosphohydrolysis and nucleoside signaling through biochemical crosstalk involving more than one cell type. We have previous demonstrated that PMNderived ATP can be "auto-hydrolyzed" to AMP through PMN surface NTPDase-1 (CD39) $(23,30)$. However, the current studies extended this work to examine whether epithelial cells might also express NTPDase surface activity. Indeed, the phosphohydrolysis of ATP (either platelet derived or native) did not require PMNs, implicating the expression NTPDase activity on the apical surface of epithelial cells. An mRNA screen revealed the expression of at least 3 ectoNTPDase family members with rank order of expression NTPDase2>>NTPDase-1>NTPDase-8. Attempts to target these molecules with shRNA have proven difficult. Differentiated, chloride-secreting epithelia are famously difficult to transfect: specific reagents to study these various family members (e.g., selective inhibitors) have not become available, and therefore, the extent to which they could be studied at the protein level has been limited. Nonetheless, a nonselective polyoxometalate NTPDase inhibitor (POM-1) confirmed the existence of significant NTPDase activity on the apical surface of the epithelium. One family member, NTPDase-1, also called CD39, has been extensively studied on vascular endothelial cells (27). This molecule is thought to function primarily in a thromboregulatory manner to modulate platelet aggregation at the vascular surface. Moreover, we have shown that this molecule regulates the rate of Ado generation at the vascular interface (22) and thus may be immunoregulatory. Under such conditions, this first-step reaction results in the liberation of AMP, and further metabolism of AMP to Ado requires the contribution of $\mathrm{CD} 73$ activity as a means to generate Ado. The expression pattern and function of CD73 are better understood than those of the NTPDases, and CD73 is highly expressed on endothelial cells, epithelial cells, and lymphocytes (26). Thus, the findings of apically expressed NTPDase(s) identify this molecule as a regulatory control point, requiring close spatial relationship to surface-expressed CD73 for Ado-mediated signaling pathways.

The epithelium normally functions to provide a selective barrier to luminal contents and to provide vectoral ion transport, the basis of water movement across the epithelium (40). We demonstrate here that luminal ATP provides a strong driving force for water movement into the mucosal lumen. Indeed, the use of an intestinal ligation model revealed prominent vectoral water transport in response to luminal ATP. Further studies using $C D 73^{-/-}$mice (25) provided definitive evidence that such responses require the apical metabolism of ATP to Ado. These animals show no outward or obvious signs of inflammation at baseline $(25,41)$, and therefore we are confident that the differences in water transport do not reflect differences resulting from underlying inflammation. Thus, we can only surmise that a physiological function for such a pathway is the "flushing" of potentially noxious components from the luminal surface during an ongoing inflammatory response. Some evidence suggests that such a mechanism may be protective in mucosal disease. For example, Asfaha et al. used a murine colitis model and demonstrated that at 6 weeks after colitis induction, barrier function defects had resolved but colonic secretory dysfunction persisted (42). These findings paralleled increased bacterial translocation and increased colonic aerobes, suggesting that prolonged secretory impairment may underlie the chronic nature of enteropathies such as IBD.
In summary, these results define a previously unappreciated metabolic pathway prevalent at the mucosal interface during inflammation. We show here that PMN- and platelet-derived ATP selectively activates epithelial ion transport through metabolism of ATP to Ado. Central to this pathway is the coordinated metabolism of extracellular ATP by ecto-nucleotidases localized on the apical epithelial membrane.

\section{Methods}

Isolation of neutrophils. PMNs were freshly isolated from whole blood obtained by venipuncture from volunteers and anticoagulated with acid citrate/dextrose, as described previously (43). Prior to blood donation, all volunteers signed informed consent statements according to existing protocols approved by the Colorado Multi-Institutional Review Board. Briefly, platelets, plasma, and mononuclear cells were removed by aspiration following centrifugation through a double layer of Histopaque-T1077 and Histopaque-T1119 (Sigma-Aldrich) at $500 \mathrm{~g}$ for 30 minutes at $22^{\circ} \mathrm{C}$. PMNs were found at the interface between T1077 and T1119. Residual erythrocytes were removed by lysis in cold $\mathrm{NH}_{4} \mathrm{Cl}$ buffer. Remaining cells were greater than $97 \%$ PMNs as assessed by microscopic evaluation. PMNs were studied within 2 hours of their isolation.

Isolation of platelets. Platelet-rich plasma was obtained from whole blood anticoagulated with acid/dextrose following centrifugation at $150 \mathrm{~g}$ for 15 minutes at $22^{\circ} \mathrm{C} \mathrm{(31).} \mathrm{Numbers} \mathrm{of} \mathrm{platelets} \mathrm{were} \mathrm{assessed} \mathrm{by} \mathrm{microscopic}$ evaluation. One milliliter of platelet-rich plasma was resuspended in $5 \mathrm{ml}$ HBSS minus and centrifuged at $1,400 \mathrm{~g}$ for 10 minutes at $4^{\circ} \mathrm{C}$. Platelets were studied within 2 hours of their isolation.

Immunohistochemical localization of PMNs and platelets. Sections from archived human tissue from patients definitively diagnosed with IBD were obtained under research protocols approved by the Colorado MultiInstitutional Review Board or the University of Calgary Conjoint Ethics Committee. Paraffin-embedded tissues were sectioned and H\&E stained by IHCtech. For fluorescence immunohistochemistry, sections were rehydrated and subjected to heat-mediated antigen retrieval, according to standard protocols. Autofluorescence was quenched as described previously (44). Nonspecific binding was blocked with a mixture of $5 \%$ normal serum and $1 \%$ IgG-free BSA in PBS. Primary antibodies used were monoclonal anti-CD41 at 1:50 (Abcam; clone 283.16B7) and prediluted polyclonal anti-MPO used neat (Abcam; ab15484). Secondary antibodies used were anti-rabbit Alexa Fluor-488 and anti-mouse Alexa Fluor-555 (Invitrogen) at a 1:100 dilution. Nuclei were counterstained with DAPI, and slides were mounted with ProLong antifade gold (Invitrogen). Sections were imaged at 100x oil immersion using a Zeiss AxioImager A1 with an AxioCam MRc5.

Preparation of activated PMNs and PMN supernatants. Freshly isolated PMNs $\left(10^{6}\right.$ cells $/ \mathrm{ml}$ in HBSS with $10^{-6} \mathrm{M}$ fMLP) were incubated as described previously (45). Activated PMNs were then either immediately used in chloride secretion experiments or pelleted $\left(1,000 \mathrm{~g}\right.$ for 20 seconds, $\left.4^{\circ} \mathrm{C}\right)$ and supernatants filtered $(0.45 \mu \mathrm{m}$; Phenomenex). For initial experiments isolating active PMN fractions, $10^{6} \mathrm{PMNs} / \mathrm{ml}$ were activated, cells were removed by pelleting, and supernatants were filtered $(0.2 \mu \mathrm{m})$. Resultant cell-free supernatants were resolved by HPLC and identified by their chromatographic behavior as described previously (30).

Preparation of activated platelets. To measure the time course of ATP release from platelets, $10^{6}$ platelets were activated with collagen for indicated periods of time, supernatants were collected, and ATP content was quantified using CHRONO-LUME reagent (Chrono-log Corp.). Luciferase activity was assessed on a luminometer (Turner Designs) and compared with internal ATP standards.

$P M N$ transmigration and platelet translocation assays. Migration assays were performed in the presence of platelets. Briefly, $10^{6}$ PMNs were 
added together with $6 \times 10^{6}$ platelets to the upper chambers of Transwell inverts in which T84 cell monolayers were plated on the opposing side. A chemotactic gradient was established by adding $1 \mu \mathrm{M}$ fMLP to the lower chambers. PMN transmigration studies were performed at $37^{\circ} \mathrm{C}$ for 90 minutes in the presence of MHC-1, CD11b, or CD47 antibodies or vehicle. Transmigrated cells were BCECF labeled and evaluated for fluorescence in the above-mentioned conditions.

Cell culture. Human intestinal epithelial cells (T84) were grown on collagen-coated permeable supports to confluent monolayers that exhibited a steady-state transepithelial resistance. Monolayers were thoroughly washed in HBSS and placed in apical and basolateral baths of HBSS during all experiments. All experiments were carried out in a $37^{\circ} \mathrm{C}$ room to ensure ideal testing conditions. To measure agonist-stimulated Isc's, transepithelial potentials, and resistance, a commercially available voltage clamp (Iowa Dual Voltage Clamps, Bioengineering, University of Iowa) interfaced with an equilibrated pair of calomel electrodes was used, as described in detail elsewhere (19) Sodium polyoxotungstate, a water-soluble polyoxometalate (POM-1, $\mathrm{Na}_{6}\left[\mathrm{H}_{2} \mathrm{~W}_{12} \mathrm{O}_{40}\right]$ ), was a generous gift from C.E. Müller, Pharmaceutical Institute, Pharmaceutical Chemistry (Bonn, Germany).

RNA isolation and transcriptional analysis. RNA was isolated and cDNA synthesized as described previously (10). Potential contaminating genomic DNA was digested using DNA-free (Ambion). Semiquantitative and realtime PCR were performed using increasing numbers of cycles of $95^{\circ} \mathrm{C}$ for 45 seconds, $59^{\circ} \mathrm{C}$ for 35 seconds, and $72^{\circ} \mathrm{C}$ for 45 seconds and a final extension time of 7 minutes (10). The following primers were used to quantify ecto-NTPDase activity on intestinal epithelial cells: E-NTPDase-1 forward, $5^{\prime}$-AGCAGCTGAAATATGCTGGC-3' and reverse, $5^{\prime}$-GAGACAGTATCTGCCGAAGTCC-3'; E-NTPDase-2 forward, 5'-GCCTCTACCCAGATCACTTTTG-3' and reverse, 5'-AGCACTTGGGTGGAAAAGC-3'; E-NTPDase- 3 forward, $5^{\prime}$-AGTTTCTGGCAATGCTCCTG- 3 ' and reverse, 5'-GGTCCCCAGTTCCTTCAAA-3'; E-NTPDase-8 forward, 5'-CCTCTACGGCTCCGACTACA-3' and reverse, 5'-TTGCCTGTCCCTTCAACTGT-3'. Transcript levels and fold change in mRNA were determined as described previously (30). Intron-overlapping primer pairs for NTPDase-2, -3 , and -8 were designed using Primer3 software (http://frodo.wi.mit.edu/). Primer properties and secondary structures including hairpins, self-dimers, and cross-dimers were evaluated in a second step using NetPrimer software (http://www.premierbiosoft.com/netprimer). Primer sets contained $10 \mathrm{pM}$ each of the sense primer and the antisense primer. The primer set was amplified using increasing numbers of cycles of $94^{\circ} \mathrm{C}$ for 1 minute, $59.5^{\circ} \mathrm{C}$ for 1 minute, $72^{\circ} \mathrm{C}$ for 1 minute, and a final extension of $72^{\circ} \mathrm{C}$ for 7 minutes. Samples were controlled for $\beta$-actin using the following primers: sense 5'-ACTGGAACGGTGAAGGTGACAG-3', antisense 5'-GGTGGCTTTTAGGATGGCAAG-3'.

Measurement of surface NTPDase enzyme activity. We assessed NTPDase surface enzyme activity as described previously (46) by quantifying the conversion of etheno-ATP (E-ATP) to etheno-AMP (E-AMP). Briefly, HBSS (with or without addition of the NTPDase inhibitor POM-1; $10 \mu \mathrm{M}$ ) was added to T84 epithelial monolayers on a 6-well plates. After 10 minutes, E-ATP (final concentration, $100 \mu \mathrm{M}$ ) was added. Samples were taken at indicated time- points, removed, acidified to $\mathrm{pH} 3.5$ with $\mathrm{HCl}$, spun $(10,000 \mathrm{~g}$ for 20 seconds, $\left.4{ }^{\circ} \mathrm{C}\right)$, filtered $(0.45 \mu \mathrm{m})$, and frozen $\left(-80^{\circ} \mathrm{C}\right)$ until analysis via reverse-phase HPLC. The ratio of E-ATP (Molecular Probes; Invitrogen) to E-AMP (SigmaAldrich) was measured with a $0 \%-50 \%$ methanol $/ \mathrm{H}_{2} \mathrm{O}$ gradient mobile phase ( $2 \mathrm{ml} / \mathrm{min}$ over 10 minutes). Absorbance was measured at $260 \mathrm{~nm}$, and ultraviolet absorption spectra were obtained at chromatographic peaks. NTPDase activity was expressed as percent E-ATP conversion in this time frame.

In vivo intestinal secretion model. After a fasting period of 12 hours, mice were anesthetized using sodium pentobarbital $(50 \mu \mathrm{g} / \mathrm{mg})$ and mechanically ventilated for 1.5 hours. A small laparotomy incision was made, and a stool-free 2- to 3-cm-long segment of the small intestine was localized. A 3-0 silk suture (Ethicon Endo-Surgery Inc., Johnson \& Johnson Health Care) was placed and tightened around the middle of the chosen segment, thereby not affecting the arterial blood supply. A second suture was placed more distal from the first suture, thereby creating a loop of $1-1.5 \mathrm{~cm}$ in length. A 22-gauge catheter was inserted over the suture into the lumen through a small incision distal to the loop. After injection of $100 \mu \mathrm{l}$ vehicle solution, the second suture was instantly tightened and the catheter removed. The same procedure was performed within the adjacent proximal segment with a $1-\mathrm{mM}$ ATP solution. The bowel was placed back in the peritoneal cavity, and the laparotomy incision was closed. Mice were euthanized 1 hour later and the colonic loops excised. Wet/dry ratios were then calculated as percentage of water increase/decrease after drug injection compared with vehicle as described previously (47). Where indicated, mice deficient in $C D 73$ were generated, their genotypes validated, and characterized as described previously (25). Original $C D 73^{-/-}$breeding stock was kindly provided by Linda Thompson (Oklahoma Medical Research Foundation, Oklahoma City, Oklahoma, USA). Littermate control mice were matched according to sex, age, and weight. This protocol was in accordance with NIH guidelines for use of live animals and was approved by the Institutional Animal Care and Use Committee at the University of Colorado.

Statistics. All experiments were performed at least in triplicate and results expressed as mean \pm SEM. Comparisons between multiple groups were made using ANOVA and between 2 groups using 2-tailed Student's $t$ test; $P<0.05$ was considered significant.

\section{Acknowledgments}

This work was supported by NIH grants HL60569 and DK50189. The authors acknowledge Linda Thompson for providing the original breeding stock of CD73-deficient mice and Christa E. Müller for kindly providing the NTPDase inhibitor POM-1.

Received for publication April 7, 2008, and accepted in revised form September 3, 2008.

Address correspondence to: Sean P. Colgan, Mucosal Inflammation Program, University of Colorado Health Science Center, Biochemistry Research Building, Room 702, 4200 E. 9th Avenue, Denver, Colorado 80262, USA. Phone: (303) 315-1064 or (303) 315-1068; Fax: (303) 315-1121; E-mail: sean.colgan@ucdenver.edu.
1. Nikolaus, S., and Schreiber, S. 2007. Diagnostics of inflammatory bowel disease. Gastroenterology. 133:1670-1689.

2. Danese, S., de la Motte, C., and Fiocchi, C. 2004. Platelets in inflammatory bowel disease: clinical, pathogenic, and therapeutic implications. Am.J. Gastroenterol. 99:938-945.

3. Zarbock, A., Polanowska-Grabowska, R.K., and Ley, K. 2007. Platelet-neutrophil-interactions: linking hemostasis and inflammation. Blood Rev. 21:99-111.

4. Vowinkel, T., et al. 2007. Mechanisms of platelet and leukocyte recruitment in experimental colitis. Am.J. Physiol. Gastrointest. Liver Physiol. 293:G1054-G1060.

5. Serhan, C.N., et al. 2007. Resolution of inflammation: state of the art, definitions and terms. FASEBJ. 21:325-332.

6. Burnstock, G. 2002. Potential therapeutic targets in the rapidly expanding field of purinergic signalling. Clin. Med. 2:45-53.

7. Wang, T.F., and Guidotti, G. 1996. CD39 is an ecto(Ca2+,Mg2+)-apyrase. J. Biol. Chem. 271:9898-9901.

8. Mizumoto, N., et al. 2002. CD39 is the dominant Langerhans cell-associated ecto-NTPDase: modu- latory roles in inflammation and immune responsiveness. Nat. Med. 8:358-365.

9. Gendron, F.P., et al. 2002. Purine signaling and potential new therapeutic approach: possible outcomes of NTPDase inhibition. Curr. Drug Targets. 3:229-245.

10. Qawi, I., and Robson, S.C. 2000. New developments in anti-platelet therapies: potential use of CD39/ vascular ATP diphosphohydrolase in thrombotic disorders. Curr. Drug Targets. 1:285-296.

11. Robson, S.C., Sevigny, J., and Zimmermann, H. 2006. The E-NTPDase family of ectonucleotidases: 
structure function relationships and pathophysiological significance. Purinergic Signal. 2:409-430.

12. Linden, J. 2001. Molecular approach to adenosine receptors: receptor-mediated mechanisms of tissue protection. Annu. Rev. Pharmacol. Toxicol. 41:775-787

13. Minamino, T., et al. 1996. Cardioprotection due to preconditioning correlates with increased ecto-5' nucleotidase activity. Am. J. Physiol. 270:H238-H244.

14. Hasko, G., and Cronstein, B.N. 2004. Adenosine: an endogenous regulator of innate immunity. Trends Immunol. 25:33-39.

15. Strohmeier, G.R., Reppert, S.M., Lencer, W.I., and Madara, J.L. 1995. The A2b adenosine receptor mediates cAMP responses to adenosine receptor agonists in human intestinal epithelia. J. Biol. Chem. 270:2387-2394.

16. Chin, A.C., and Parkos, C.A. 2007. Pathobiology of neutrophil transepithelial migration: implications in mediating epithelial injury. Annu. Rev. Pathol. 2:111-143.

17. Lee, W.Y., Chin, A.C., Voss, S., and Parkos, C.A 2006. In vitro neutrophil transepithelial migration. Methods Mol. Biol. 341:205-215.

18. Parkos, C.A., et al. 1996. CD 47 mediates postadhesive events required for neutrophil migration across polarized intestinal epithelia. J. Cell Biol. 132:437-450

19. Parkos, C.A., Delp, C., Arnaout, M.A., and Madara, J.L. 1991. Neutrophil migration across a cultured intestinal epithelium: dependence on a CD11b/ CD18-mediated event and enhanced efficiency in the physiologic direction. J. Clin. Invest. 88:1605-1612.

20. Madara, J.L., et al. 1992. Cl- secretion in a model intestinal epithelium induced by a neutrophilderived secretagogue. J. Clin. Invest. 89:1938-1944.

21. Madara, J.L., et al. 1993. 5'-adenosine monophosphate is the neutrophil-derived paracrine factor that elicits chloride secretion from T84 intestinal epithelial cell monolayers. J. Clin. Invest. 91:2320-2325.

22. Lennon, P.F., Taylor, C.T., Stahl, G.L., and Colgan, S.P. 1998. Neutrophil-derived 5'-adenosine monophosphate promotes endothelial barrier function via CD73-mediated conversion to adenosine and endothelial $\mathrm{A}_{2 \mathrm{~B}}$ receptor activation. J. Exp. Med. 188:1433-1443.
23. Eltzschig, H.K., et al. 2006. ATP release from activated neutrophils occurs via connexin 43 and modulates adenosine-dependent endothelial cell function. Circ. Res. 99:1100-1108.

24. Strohmeier, G.R., et al. 1997. Surface expression, polarization, and functional significance of CD73 in human intestinal epithelia. J. Clin. Invest. 99:2588-2601.

25. Thompson, L.F., et al. 2004. Crucial role for ecto5 -nucleotidase (CD73) in vascular leak during hypoxia. J. Exp. Med. 200:1395-1405.

26. Colgan, S.P., Eltzschig, H.K., Eckle, T., and Thompson, L.F. 2006. Physiologic roles for ecto-5'-nucleotidase (CD73). Purinergic Signalling. 2:351-360.

27. Robson, S.C., et al. 2005. Ectonucleotidases of CD39 family modulate vascular inflammation and thrombosis in transplantation. Semin. Thromb. Hemost. 31:217-233.

28. Vaishnava, S., and Hooper, L.V. 2007. Alkaline phosphatase: keeping the peace at the gut epithelial surface. Cell Host Microbe. 2:365-367.

29. Müller, C.E., et al. 2006. Polyoxometalates - a new class of potent ecto-nucleoside triphosphate diphosphohydrolase (NTPDase) inhibitors. Bioorg. Med. Chem. Lett. 16:5943-5947.

30. Eltzschig, H.K., et al. 2003. Coordinated adenine nucleotide phosphohydrolysis and nucleoside signaling in posthypoxic endothelium: role of ectonucleotidases and adenosine $\mathrm{A}_{2 \mathrm{~B}}$ receptors. J. Exp. Med. 198:783-796.

31. Marcus, A.J., et al. 1984. Production of arachidonic acid lipoxygenase products during platelet-neutrophil interactions. Clin. Physiol. Biochem. 2:78-83.

32. Marcus, A.J., et al. 1982. Formation of leukotrienes and other hydroxy acids during platelet-neutrophil interactions in vitro. Biochem. Biophys. Res. Commun. 109:130-137.

33. Levy, B.D., et al. 1993. Agonist-induced lipoxin $\mathrm{A}_{4}$ generation: detection by a novel lipoxin $\mathrm{A}_{4}$ ELISA. Lipids. 28:1047-1053.

34. Serhan, C.N., and Sheppard, K.A. 1990. Lipoxin formation during human neutrophil-platelet interactions. Evidence for the transformation of leukotriene A4 by platelet 12 -lipoxygenase in vitro. J. Clin. Invest. 85:772-780.

35. Novak, I. 2003. ATP as a signaling molecule: the exocrine focus. News Physiol. Sci. 18:12-17.

36. Goodenough, D.A., and Paul, D.L. 2003. Beyond the gap: functions of unpaired connexon channels. Nat. Rev. Mol. Cell Biol. 4:285-294.

37. Bermudez-Fajardo, A., Yliharsila, M., Evans, W.H., Newby, A.C., and Oviedo-Orta, E. 2007. CD4+ T lymphocyte subsets express connexin 43 and establish gap junction channel communication with macrophages in vitro. J. Leukoc. Biol. 82:608-612.

38. Eugenin, E.A., Gonzalez, H.E., Sanchez, H.A., Branes, M.C., and Saez, J.C. 2007. Inflammatory conditions induce gap junctional communication between rat Kupffer cells both in vivo and in vitro. Cell. Immunol. 247:103-110.

39. Linden, J. 2005. Adenosine in tissue protection and tissue regeneration. Mol. Pharmacol. 67:1385-1387.

40. McCole, D.F., and Barrett, K.E. 2007. Varied role of the gut epithelium in mucosal homeostasis. Curr. Opin. Gastroenterol. 23:647-654.

41. Louis, N.A., et al. 2008. Control of IFN-\{alpha\}A by CD73: implications for mucosal inflammation. J. Immunol. 180:4246-4255.

42. Asfaha, S., et al. 2001. Persistent epithelial dysfunction and bacterial translocation after resolution of intestinal inflammation. Am. J. Physiol. Gastrointest. Liver Physiol. 281:G635-G644.

43. Henson, P., and Oades, Z.G. 1975. Stimulation of human neutrophils by soluble and insoluble immunoglobulin aggregates. J. Clin. Invest. 56:1053-1061.

44. Baschong, W., Suetterlin, R., and Laeng, R.H. 2001. Control of autofluorescence of archival formaldehyde-fixed, paraffin-embedded tissue in confocal laser scanning microscopy (CLSM). J. Histochem. Cytochem. 49:1565-1572.

45. Campbell, E.L., et al. 2007. Resolvin E1 promotes mucosal surface clearance of neutrophils: a new paradigm for inflammatory resolution. FASEB J. 21:3162-3170.

46. Synnestvedt, K., et al. 2002. Ecto-5'-nucleotidase (CD73) regulation by hypoxia-inducible factor-1 (HIF-1) mediates permeability changes in intestinal epithelia. J. Clin. Invest. 110:993-1002

47. Khoury, J., Ibla, J.C., Neish, A.S., and Colgan, S.P. 2007. Antiinflammatory adaptation to hypoxia through adenosine-mediated cullin-1 deneddylation. J. Clin. Invest. 117:703-711. 